\title{
HOPF BIFURCATION IN THE PRESENCE OF SYMMETRY
}

\author{
BY MARTIN GOLUBITSKY ${ }^{1,2}$ AND IAN STEWART ${ }^{2}$
}

In this note we state a generalization of the Hopf bifurcation theorem to differential equations with symmetry. We state the results for ordinary differential equations although they apply, via standard reduction techniques (see Marsden and McCracken [1976]), to certain partial differential equations as well. Consider the ordinary differential equation

$$
d z / d t+F(z, \lambda)=0 \quad\left(F: \mathbf{R}^{n} \times \mathbf{R} \rightarrow \mathbf{R}^{n} \text { is } C^{\infty}\right),
$$

where $F(0, \lambda)=0$ and $F$ commutes with an (orthogonal) action of a compact Lie group $\Gamma$ on $V=\mathbf{R}^{n}$; that is, $F(\gamma x, \lambda)=\gamma F(x, \lambda)$ for $\gamma \in \Gamma$. Assume $\left.d F\right|_{0,0}$ has pure imaginary eigenvalues. The symmetry can force these eigenvalues to have high multiplicity and the standard Hopf theorem does not apply. Despite this degeneracy, the symmetry can also force the occurrence of a branch of periodic solutions to (1).

Interactions between Hopf-type bifurcation and symmetry have been studied previously by several authors. Ruelle $[\mathbf{1 9 7 3}]$ deals mainly with bifurcations of mappings, Schecter [1976] analyzes the continuous case. Rand [1982] and Renardy [1982] mainly consider bifurcations to tori from rotating waves. Schecter [1976], Bajaj [1982], Van Gils [1984], and Chossat and Iooss [1984] all consider the example $\Gamma=\mathbf{O}(2)$. Our approach differs from these by emphasizing the general role of isotropy subgroups in determining the occurrence of branches.

These ideas prove useful in studying Taylor-Couette flow of a fluid between coaxial rotating cylinders. See Chossat and Iooss [1984] and Golubitsky and Stewart [1984b]. Other potential applications include systems of identical coupled chemical oscillators (see Alexander and Auchmuty [1984]).

For $x \in V$ define the isotropy group $\Sigma_{x}=\{\sigma \in \Gamma \mid \sigma x=x\}$. Let $\Sigma \subseteq \Gamma$ and define the fixed-point subspace $V^{\Sigma}=\{y \in V \mid \sigma y=y$ for all $\sigma \in \Sigma\}$. Notice that $F$ maps $V^{\Sigma}$ to itself.

In order for $\left.d F\right|_{0,0}$ to have pure imaginary eigenvalues, the representation of $\Gamma$ on $V$ must satisfy certain conditions. There are two 'simplest' cases:

(a) The action of $\Gamma$ on $V$ is irreducible but not absolutely irreducible.

(b) $V=W \oplus W$, where $\Gamma$ acts absolutely irreducibly on $W$ and by the diagonal action on $W \oplus W$.

Henceforth we assume (b) holds. If $\left.d F\right|_{0,0}$ has pure imaginary eigenvalues then we may assume, without loss of generality, that $\left.d F\right|_{0,0}=\left(\begin{array}{cc}0 & -I \\ I & 0\end{array}\right)$. The eigenvalues of $\left.d F\right|_{0, \lambda}$ are $\sigma(\lambda) \pm i \phi(\lambda)$, each of multiplicity $\operatorname{dim} W=n / 2$.

Received by the editors January 17, 1984 and, in revised form, March 30, 1984.

1980 Mathematics Subject Classification. Primary 58F22; Secondary 58F14.

${ }^{1}$ Research supported in part by NSF Grant MCS-8101580.

${ }^{2}$ Research supported in part by a grant from NASA-Ames, NAG 2-279.

(c) 1984 American Mathematical Society $0273-0979 / 84 \$ 1.00+\$ .25$ per page 
We assume the transversality condition $\sigma^{\prime}(0) \neq 0$. By scaling time we may assume the eigenvalues are $\pm i$ at $\lambda=0$.

To motivate our main result, we note that if $\Sigma \subseteq \Gamma$ is an isotropy group with $\operatorname{dim} W^{\Sigma}=1$, then there exists a branch of periodic $\Sigma$-invariant solutions to (1), of period near $2 \pi$, bifurcating at $\lambda=0$. Indeed, restrict $F$ to $(W \oplus W)^{\Sigma}$, which has dimension 2 . The eigenvalues of $\left.d F\right|_{0,0}$ become simple: apply Hopf.

Periodicity induces a further temporal $\mathbf{S}^{1}$ symmetry by a shift of phase. (Here $\mathbf{S}^{1}$ is the circle group.) Define an action of $\Gamma \times \mathbf{S}^{1}$ on $W \oplus W$ by identifying $W \oplus W$ with $W \otimes_{\mathbf{R}} \mathbf{C}$ and setting $(\gamma, \theta) w \otimes z=\gamma w \otimes e^{i \theta} z$. Apply the Liapunov-Schmidt reduction proof of the Hopf Theorem (Hale [1978], Golubitsky and Langford [1981]), introducing a period-scaling parameter $\tau$ as usual. This procedure leads to a reduced bifurcation equation $g(x, \lambda, \tau)=0$, where $g:(W \otimes \mathbf{C}) \times \mathbf{R} \times \mathbf{R} \rightarrow W \otimes \mathbf{C}$ commutes with the $\Gamma \times \mathbf{S}^{1}$ action on $W \otimes \mathbf{C}$. Sattinger $[\mathbf{1 9 8 3}]$ has observed that the Liapunov-Schmidt procedure leads to $g$ having $\Gamma \times \mathbf{S}^{1}$ symmetry.

Our main result, which is the periodic analogue of an equivariant branching lemma of Cicogna [1981], is:

EQUIVARIANT HOPF THEOREM. Let $\Sigma \subseteq \Gamma \times \mathbf{S}^{1}$ be an isotropy group with $\operatorname{dim}(W \otimes \mathbf{C})^{\Sigma}=2$. Then there exists a branch of periodic $\Sigma$-invariant solutions to (1), of period near $2 \pi$, bifurcating at $\lambda=0$.

The idea of the proof is to analyse the structure of $g \mid(W \otimes \mathbf{C})^{\Sigma} \times \mathbf{R} \times \mathbf{R}$ using symmetry arguments.

Note. If $(\gamma, \theta)$ is in the isotropy subgroup of a solution $z(t)$ to $(1)$, then

$$
z(t)=\gamma z(t+\theta) \text {. }
$$

Therefore $z$ has a mixture of spatial $(\gamma)$ and temporal $(\theta)$ symmetry.

Chow, Mallet-Paret and Yorke [1978] use the Fuller index to prove the existence of a branch of periodic solutions to $(1)$ when $\sigma^{\prime}(0) \neq 0$. Using our methods we can prove the existence of many periodic solutions branches each with prescribed symmetries.

EXAMPLE 1. $\Gamma=\mathbf{O}(2)$. Let $\Gamma$ act on $\mathbf{R}^{2}=W$ as usual. There are two conjugacy classes of isotropy groups $\Sigma$ with a 2-dimensional fixed-point subspace. One is given by the reflectional symmetry in $\mathbf{O}(2)$. The other is isomorphic to $\mathbf{S O}(2)$ and consists of all $(\theta,-\theta) \in \mathbf{O}(2) \times \mathbf{S}^{1}$. Such a solution $z(t)$ satisfies $z(t+\theta)=R_{\theta} z(t)$, where $R_{\theta}$ is rotation. This is a rotating wave; see Auchmuty $[\mathbf{1 9 7 9}]$. We thus recover the results of Schecter [1976]. Note how the symmetry under $\Sigma$ identifies the solution as a rotating wave.

For general $\Gamma$, every isotropy subgroup $\Sigma$ of $\Gamma \times \mathbf{S}^{1}$ is of the form $\{(h, \theta(h))$ $\mid h \in H \subset \Gamma\}$, where the twist $\theta: H \rightarrow \mathbf{S}^{1}$ is a homomorphism. Let $K=$ $\operatorname{ker}(\theta)=\Sigma \cap \Gamma$. Say that $\Sigma$ is spatial if $\operatorname{dim}(W \otimes \mathbf{C})^{K}=2$. The spatial subgroups were covered by the simple Hopf theorem above. Nontrivially twisted groups $\Sigma$ give rise to solutions which combine spatial symmetry $K$ with a generalized rotating wave symmetry $H / K$.

EXAMPLE 2. $\Gamma=\mathbf{O}(3)$. By using ideas from representation theory (cf. Ihrig and Golubitsky [1984]) we have classified all possible $\Sigma$ satisfying $\operatorname{diam}(W \otimes \mathbf{C})^{\Sigma}=2$ when $\Gamma=\mathbf{O}(3)$ acts on spherical harmonics of order 
$l$ for all $l$. For small $l$ we tabulate those results here:

$l$

1

2
$H$

$$
\begin{aligned}
& \mathbf{O}(2), \mathbf{S O}(2) \\
& \mathbf{S O}(2) \\
& \mathbf{O}(2), \mathbf{D}_{2}, \mathbf{D}_{4} \\
& \mathbf{T} \\
& \mathbf{S O}(2) \text { (twice) } \\
& \mathbf{O}(2), \mathbf{S O}(2) \text { octahedral } \\
& \quad \text { group } \mathbf{O}, \mathbf{D}_{2}, \mathbf{D}_{3}
\end{aligned}
$$

SO(2) (three times) (spatial)

(rotating wave $(\theta,-\theta)$ )

(spatial)

( $\mathbf{Z}_{3}$-twisted tetrahedral)

(rotating waves $(\theta,-\theta),(\theta,-2 \theta)$ )

(spatial)

(rotating waves $(\theta,-\theta),(\theta,-2 \theta)$,

$$
(\theta,-3 \theta))
$$

Note in particular the $\mathbf{Z}_{3}$-twisted tetrahedral group. Such twistings of $\mathbf{T}$ occur for $l=2,4,5,6,7,9$ precisely.

By putting (1) into normal form, that is, assuming $F$ commutes with $\Gamma \times \mathbf{S}^{\mathbf{1}}$, we can describe the orbital stability of the bifurcating periodic solutions in specific cases, including $\Gamma=\mathbf{O}(2)$. We show that for a vector field in normal form, stability is determined by the eigenvalues of the reduced mapping $g$ (compare Kielhöfer and Lauterbach [1983]). Subcritical solutions are unstable, but supercritical solutions need not be stable, even for $\mathbf{O}(2)$.

By an extension argument involving hardly any computations we show that the case of $\mathbf{O}(n)$ acting on $\mathbf{R}^{n}$ exactly parallels that for $\mathbf{O}(2)$. a].

Further results and detailed proofs are in Golubitsky and Stewart [1984

\section{REFERENCES}

J. Alexander and J. F. G. Auchmuty [1984], Bifurcation of phase-locked solutions of coupled oscillators (in preparation).

J. F. G. Auchmuty [1979], Bifurcating waves, Ann. New York Acad. Sci. 316, 263-278.

A. K. Bajaj [1982], Bifurcating periodic solutions in rotationally symmetric systems, SIAM J. Appl. Math. 42, 1078-1098.

P. Chossat and G. Iooss [1984], Primary and secondary bifurcation in the Couette-Taylor problem, Nice (preprint).

S. N. Chow, J. Mallet-Paret and J. Yorke [1978], Global Hopf bifurcation from a multiple eigenvalue, Nonlinear Anal. 2, 753-763.

G. Cicogna [1981], Symmetry breakdoun for bifurcations, Lett. Nuovo. Cimento (2) 31, 600-602.

M. Golubitsky and W. F. Langford [1981], Classification and unfoldings of degenerate Hopf bifurcations, J. Differential Equations 41, 375-415.

M. Golubitsky and I. N. Stewart [1984a], Hopf bifurcation in the presence of symmetry, Arch. Rational Mech. Anal. (to appear). mitted).

[1984b], Symmetry and stability in Taylor-Couette flow, SIAM J. Math. Anal. (sub-

J. K. Hale [1978], Nonlinear analysis and mechanics: Heriot-Watt Symposium, Vol. I (R. J. Knopf, ed.), Res. Notes in Math., Vol. 17, Pitman, San Francisco, pp. 59-157.

E. Ihrig and M. Golubitsky [1984], Pattern selection with $\mathbf{O}(3)$ symmetry, Phys. D (to appear).

H. Kielhöfer and R. Lauterbach [1983], On the principle of reduced stability, J. Funct. Anal. 53, 99-111. 
J. E. Marsden and M. McCracken [1976], The Hopf bifurcation and its applications, Appl. Math. Sci. 19, Springer, New York.

D. Rand [1982], Dymamics and symmetry: Predictions for modulated waves in rotating fluids, Arch. Rational Mech. Anal. 79, 1-38. 84.

M. Renardy [1982], Bifurcation from rotating waves, Arch. Rational Mech. Anal. 75, 49-

D. Ruelle [1973], Bifurcation in the presence of a symmetry group, Arch. Rational Mech. Anal. 51, 136-152.

D. H. Sattinger [1983], Branching in the presence of symmetry, CBMS Regional Conf. Ser. in Appl. Math., no. 40, SIAM, Philadelphia.

S. Schecter [1976], Bifurcations with symmetry, The Hopf bifurcation and Its Applications (J. E. Marsden and M. McCracken, eds.), Appl. Math. Sci. 19, Springer, New York, pp. 224-249.

S. A. Van Gils [1984], Some studies in dymamical system theory, Ph.D. Thesis, Vrije Univ., Amsterdam.

DEPARTMENT OF MATHEMATICS, UNIVERSITY OF HOUSTON-UNIVERSITY PARK, HOUSTON, TEXAS 77004

MATHEMATICS Institute, UNiversity OF WARWICK, COVENTRY CV4 7AL, ENGLAND 